\title{
Clinical Presentation of Acquired Hypothyroidism and Associated Disorders in Children and Adolescents at King Abdul-Aziz University Hospital in a Western Region of Saudi Arabia
}

\author{
Al-Agha $\mathrm{AE}^{1 *}$, Alshugair $\mathrm{RM}^{2}$, Aljunedi $\mathrm{WA}^{3}$ and Badakhan $\mathrm{BA}^{3}$ \\ ${ }^{1}$ Pediatric Department, King Abdul-Aziz University Hospital, Saudi Arabia \\ ${ }^{2}$ Department of Medicine, King Abdul-Aziz University, Saudi Arabia
}

${ }^{3}$ Department of Medicine, Ibn Sina National College, Saudi Arabia

\begin{abstract}
Objective: We aimed to investigate the clinical manifestations and associations of acquired hypothyroidism in children at King Abdul-Aziz University Hospital (KAUH) in the western region of Saudi Arabia.

Methods: A retrospective descriptive study was conducted in Jeddah, Saudi Arabia from January 2010 to January 2015. The study included 265 children aged between one and 18 years old with a confirmed diagnosis of acquired hypothyroidism who attended the pediatric endocrine clinic at KAUH. Data were obtained by reviewing the medical records and laboratory investigations of the patients by using KAUH's "phoenix" system.

Results: Of the 265 children diagnosed with acquired hypothyroidism, the commonest clinical presentations were short stature $(32.5 \%)$, loss of appetite $(16.2 \%)$, weight gain $(13.6 \%)$, fatigability $(12.1 \%)$, constipation $(9.8 \%)$, cold intolerance (5.3\%), and goiter (2.6\%). Vitamin D deficiency was the commonest associated disorder (present in $37 \%$ of patients) followed by type 1 diabetes mellitus (in 22.3\%) and Down syndrome (in $8.3 \%$ ).

Conclusion: Isolated short stature was the most common presentation of acquired hypothyroidism. Pediatricians should be aware of the need to screen children who present with short stature for hypothyroidism. Vitamin D deficiency was the commonest disorder associated with acquired hypothyroidism. In our setting, vitamin $D$ supplementation would be beneficial as a preventive measure.
\end{abstract}

Keywords: Acquired; Hypothyroidism; Short; Stature; Vitamin D

\section{Introduction}

Thyroid hormones play a major role in children's metabolism, growth, and pubertal maturation. Hypothyroidism is the most common disturbance of thyroid function in children [1]. Acquired hypothyroidism can be either primary (thyroid disease) or secondary to a central cause (hypothalamic-pituitary disease). Autoimmune destruction, iodine deficiency and infiltrative disease could lead to primary thyroid gland failure [2]. Iatrogenic forms of hypothyroidism occur after thyroid surgery, radioiodine therapy and neck irradiation [3]

The most common manifestation of hypothyroidism in children is decreasing growth velocity resulting in short stature [1]. Growth delay has an insidious onset and may occur several years before the occurrence of other symptoms. Common symptoms include sluggishness, lethargy, cold intolerance, constipation, dry skin, brittle hair, facial puffiness and muscle aches and pains [1]. Central hypothyroidism presents with symptoms such as headaches, visual disturbance and/or manifestations of other pituitary hormone deficiencies [1].

The most appropriate tests required to diagnose a child with hypothyroidism are serum TSH and the thyroid hormones free triiodothyronine (fT3) and free thyroxin (fT4). The combination of elevated serum TSH level with low levels of thyroid hormones indicates primary hypothyroidism, whereas a high serum TSH level with normal levels of thyroid hormones identifies subclinical hypothyroidism. A low serum fT4 with low or inappropriately normal serum TSH is associated with secondary/tertiary hypothyroidism; this necessitates further investigation of hypothalamic-pituitary insufficiency.

It is important to early diagnose and treat the acquired hypothyroidism, as it is most often associated with autoimmune dysfunction. Therefore, identification of these cases and restoration of thyroid hormone will help to restore normal sexual development and high velocity. The aim of present study was to investigate the clinical manifestations and associations of acquired hypothyroidism in children and adolescents at King Abdul-Aziz University Hospital (KAUH) in the western region of Saudi Arabia.

\section{Methods}

\section{Study setting and participants}

A retrospective descriptive study was conducted to review the clinical presentation and associated disorders of acquired hypothyroidism. Data collection took place at King Abdul-Aziz University Hospital, Jeddah, Saudi Arabia. The population was 265 children and adolescents aged one to 18 years at the time of diagnosis.

The data was obtained by reviewing the medical records of children and adolescents with acquired hypothyroidism that was being followed up at the pediatric endocrinology clinic at KAUH from January 2010 to

*Corresponding author: Abdulmoein EAl-Agha, Pediatric Department, Professor of Pediatric Endocrinology King Abdul-Aziz University Hospital, P.O.Box80215 Jeddah 21589, Saudi Arabia, Tel: 00966505590459; Fax: 96626403841,+96626408353; E-mail: aagha@kau.edu.sa

Received October 27, 2016; Accepted November 23, 2016; Published November 30, 2016

Citation: Al-Agha AE, Alshugair RM, Aljunedi WA, Badakhan BA (2016) Clinica Presentation of Acquired Hypothyroidism and Associated Disorders in Children and Adolescents at King Abdul-Aziz University Hospital in a Western Region of Saudi Arabia. J Pat Care 2: 123. doi: 10.4172/2573-4598.1000123

Copyright: (c) 2016 Al-Agha AE, et al. This is an open-access article distributed under the terms of the Creative Commons Attribution License, which permits unrestricted use, distribution, and reproduction in any medium, provided the original author and source are credited. 
Citation: Al-Agha AE, Alshugair RM, Aljunedi WA, Badakhan BA (2016) Clinical Presentation of Acquired Hypothyroidism and Associated Disorders in Children and Adolescents at King Abdul-Aziz University Hospital in a Western Region of Saudi Arabia. J Pat Care 2: 123. doi: 10.4172/25734598.1000123

January 2015. All laboratory findings were obtained by using KAUH's electronic Phoenix system. The data was collected in a questionnaire form. This data contains demographic information, anthropometric measurements, clinical presentation, presence of other associated diseases, laboratory investigations (TSH, fT4 and fT3 levels; vitamin D level; glucose level; presence of thyroid autoantibodies) and thyroid ultrasound and brain magnetic resonance imaging (MRI) findings. Ethical approval for this study was obtained from the Research Ethics Committee of KAUH.

\section{Definitions}

Primary hypothyroidism was defined as follows: serum TSH level $>5$ $\mathrm{mIU} / \mathrm{L}$ and $\mathrm{fT} 4$ level $<12 \mathrm{pmol} / \mathrm{L}$. Secondary/tertiary hypothyroidism was defined as follows: normal or low TSH level $(0.36-5.00 \mathrm{mIU} / \mathrm{L})$ and low fT4 level $(<12 \mathrm{pmol} / \mathrm{L})$. Clinical hypothyroidism was defined as a high TSH $(>5 \mathrm{mIU} / \mathrm{L})$ and low fT4 $(<12 \mathrm{pmol} / \mathrm{L})$ level, while subclinical hypothyroidism was defined as a high TSH $(>5 \mathrm{mIU} / \mathrm{L})$ and normal fT4 (12-22 pmol/L) level. The diagnosis of autoimmune thyroiditis was made based on antithyroid antibody testing: An antithyroid peroxidase antibody (anti-TPO) value $>35 \mathrm{IU} / \mathrm{mL}$ and/or antithymoglobulin antibody (anti-TG) value $>20 \mathrm{IU} / \mathrm{mL}$ confirmed the diagnosis.

Detection of T1DM was based on laboratory findings, including a fasting plasma glucose (FPG) value of $\geq 126 \mathrm{mg} / \mathrm{dL}(\geq 7.0 \mathrm{mmol} / \mathrm{L})$, random glucose value of $\geq 200 \mathrm{mg} / \mathrm{dL}(\geq 11.1 \mathrm{mmol} / \mathrm{L})$, and glycosylated hemoglobin A1c $\geq 6.5 \%$ as recommended by American Diabetes Association [4]. Vitamin D deficiency was diagnosed if the serum 25 -hydroxyvitamin D level was $<20 \mathrm{ng} / \mathrm{mL}$. Short stature was defined as a standing height $>2$ standard deviations (SD) below the mean for age, sex and race [5].

We included patients with acquired hypothyroidism aged 1-18 years old at time of diagnosis. Patients were excluded if they were older than 18 or nahy re nuoy one year of age, had incomplete data or were followed up at other medical institutions.

\section{Statistical analysis}

Descriptive statistical analysis was performed using Microsoft Excel (2013 version) and IBM SPSS Statistics, Version 21.0 (Armonk, NY: IBM Corp.). Data were entered, coded, and then analyzed. Frequency and percentages were calculated for qualitative variables including, gender, presentation, and symptoms. The mean \pm SD of quantitative data were calculated.

\section{Results}

The study included 265 children and adolescents, 152 (57.4\%) girls and $113(42.6 \%)$ boys (Table 1). The mean age at diagnosis was 8 years. Height SDS was found to be $-2.7 \pm 0.3$. A total of 132 patients $(50 \%)$ presented with symptoms suggestive of thyroid disease, $24 \%$ were detected by routine annual investigation of children with T1DM and $26 \%$ were diagnosed based on routine investigations for short stature

\begin{tabular}{|l|c|}
\hline \multicolumn{1}{|c|}{ Characteristic } & Value \\
\hline Sex & $152(57.4 \%)$ \\
\hline Female & $113(42.6 \%)$ \\
\hline Male & $3: 2.6$ \\
\hline Female/Male & $8(4.8)$ \\
\hline Age at diagnosis & $1-18$ \\
\hline Mean (SD) & \\
\hline Range & \\
\hline
\end{tabular}

Table 1: Demographic data of patients with acquired hypothyroidism.

\section{Symptoms of acquired hypothyroidism}

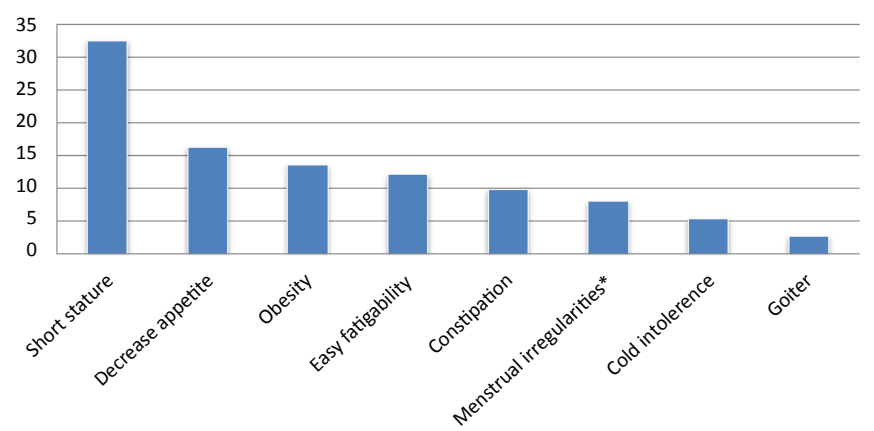

*Menstrual irregularities percentage in females older than 11 years

Figure 1: Bar chart representing symptoms of acquired hypothyroidism in relation to the percentage in the studied acquired hypothyroidism paediatric patients.

\begin{tabular}{|c|c|c|}
\hline Associated disease & Frequency & Percentage \\
\hline Vitamin D deficiency & 98 & $37 \%$ \\
\hline Type 1 diabetes mellitus & 59 & $22.3 \%$ \\
\hline Down syndrome & 22 & $8.3 \%$ \\
\hline
\end{tabular}

Table 2: Diseases associated with acquired hypothyroidism in children and adolescents with acquired hypothyroidism.

with no other manifestations of hypothyroidism. Clinical symptoms of patients with hypothyroidism are shown in Figure 1. The most common associated disease was vitamin D deficiency (Table 2). Almost all participants had primary hypothyroidism (92.5\%), while only $7.5 \%$ had secondary hypothyroidism due to pituitary adenoma/brain malformation.

\section{Discussion}

Acquired hypothyroidism is the most common abnormality of thyroid function in children and is most often caused by chronic autoimmune thyroiditis, with a prevalence of $1.2 \%$ in a six-year survey of 5,179 school children in Arizona, Utah and Nevada [6]. Normal or low levels of thyroid stimulating hormone (TSH) relative to decreased levels of thyroid hormones are characteristic of central hypothyroidism, which is often missed if physicians request measurement only of TSH levels. Acquired hypothyroidism affects both sexes; however, a female preponderance has been demonstrated in several studies.

A study of autoimmune thyroiditis conducted in 2008 showed a male-to-female ratio of 1:4.2 [7]. Moreover, a retrospective study conducted in 2007 by Demirbilek et al. reported a male-to-female ratio of $4: 6$ [8]. The reasons for this sex difference are unknown. A study conducted in 2006 demonstrated an association with chromosomal factors, such as skewed X-chromosome inactivation, while another study conducted in 1983 by Ansar Ahmed et al. found a relationship with the activity of oestrogen and progesterone which would further appear to have antagonistic influences in this particular situation $[9,10]$. No significant sex difference was observed in the present study.

Short stature is a well-recognized consequence of hypothyroidism during childhood, and can be the presenting symptom. Hence, pediatricians should be aware of the need to screen children who present with short statue for hypothyroidism. In the current study, $32.5 \%$ of the patients had short stature. In a study by Chowdhury et al., the authors reported the incidence of hypothyroidism in male and female patients with short stature to be $19 \%$ and $28 \%$, respectively 
Citation: Al-Agha AE, Alshugair RM, Aljunedi WA, Badakhan BA (2016) Clinical Presentation of Acquired Hypothyroidism and Associated Disorders in Children and Adolescents at King Abdul-Aziz University Hospital in a Western Region of Saudi Arabia. J Pat Care 2: 123. doi: 10.4172/25734598.1000123

[11]. An Indian study reported a prevalence of short stature of $45 \%$ among juvenile hypothyroid patients with height SDS $-2.9 \pm 0.9$, while in current study it found to be $-2.7 \pm 0.3 .12$ Skeletal manifestations of hypothyroidism were predominantly found in hand X-rays. In Gutch et al. study the most common finding in hand was delayed bone age with prevalence of $72 \%$ [12]. Many children with hypothyroidism have a normal growth potential after reaching euthyroid state.

Children with hypothyroidism have a decreased metabolic rate, have low levels of energy and tend to be obese. Many studies have shown high serum TSH levels in obese children and adults compared with normal weight individuals. In a study performed in Germany, TSH levels were determined in 100 obese girls and 32 normal-weight girls; the TSH and fT3 levels of the obese girls were significantly higher than those of the normal-weight girls [13]. Furthermore, in a study conducted in Germany in 2002, TSH levels were measured in 118 obese children (aged 4.5-16 years) and in 107 healthy children of normal weight; the TSH levels were significantly higher in the obese children [14]. In the present study, $13.6 \%$ of participants were obese, consistent with the above-mentioned studies.

Hypothyroidism is often associated with menstrual irregularities such as menorrhagia. A study conducted in 1964 found that $56 \%$ of hypothyroid patients had menstrual irregularities [15]. A Greek study, conducted in 1999 , showed that approximately $23.4 \%$ of hypothyroid patients had some kind of menstrual irregularity, while only $8.4 \%$ of normal controls had menstrual irregularity [16]. In the current study, we found that $7.9 \%$ of girls older than 11 years had abnormal menstruation, lower than the proportions reported in previous similar studies.

Several symptoms and signs of hypothyroidism vary according to the magnitude of thyroid hormone deficiency and the acuteness with which the deficiency develops. For example, the patient may suffer from constipation resulting from decreased gut motility; this is considered one of the commonest complaints of patients with hypothyroidism. Easy fatigability is another symptom; it occurs due to impaired respiratory function as well as hypothyroidism-related cardiovascular disease [17]. In the present study, we found that decreased appetite was a common symptom of hypothyroidism, consistent with findings of a previous study [8]. However, goiter, easy fatigability, cold intolerance and constipation were uncommon presentations in our study. A previous study investigated the clinical presentation of children and adolescents with autoimmune thyroiditis who were referred to a tertiary care center.

It indicated that the most common reasons for referral were goiter, followed by clinical symptoms of hypothyroidism. It showed that goiter was present in $77.2 \%$ of cases, fatigue in $21.6 \%$, cold intolerance in $6.9 \%$, and constipation in $5.9 \%$ of cases [7].

Children with certain chromosomal disorders, such as Down syndrome (trisomy 21), or other autoimmune disorders, such as type 1 (autoimmune) diabetes mellitus (T1DM), are at increased risk of chronic autoimmune thyroiditis. Vitamin D deficiency is an important association that should be screened for, especially in Saudi Arabian children [18-20].

Vitamin D deficiency is a global health problem. The role of vitamin $\mathrm{D}$ as an immune modulator has been recently emphasized [20]. A recent study conducted in Korea found that patients with elevated antithyroid antibodies had lower levels of serum 25(OH)D3 than those without such elevations [21]. A study conducted in 2011 showed that the prevalence of vitamin D deficiency was significantly higher in patients with autoimmune thyroid diseases than in healthy individuals ( $72 \%$ vs.
30.6\%) [22]. Moreover, a study conducted in Saudi Arabia, Alqassim region, on vitamin $\mathrm{D}$ deficiency and its associations with thyroid disease concluded that patients with hypothyroidism suffered from vitamin D deficiency [23]. In the current study we investigated diseases associated with hypothyroidism. We found that $37 \%$ of patients had vitamin D deficiency. However, this study had some limitations, notably a lack of information on nutrition and social behavior, specifically engagement in outdoor activity.

Patients with T1DM, have a higher prevalence of thyroid disorders than the normal population. The prevalence varies among different countries, ranging from $15 \%$ to $30 \%$ [24-27]. The association between T1DM and autoimmune thyroid disease has long been recognized. In this study $23.5 \%$ of patients with hypothyroidism had T1DM. A case control study was performed in Denmark in 1999 to study the association between T1DM and autoimmune thyroid disease. The authors concluded that a large proportion of diabetic children and adolescents with a relatively short duration of diabetes had markers of thyroid autoimmune disease [28]. A study conducted in 1990 assessing thyroid screening in children with T1DM showed that 19\% of patients with T1DM had positive thyroid-specific autoantibodies. The authors, therefore, recommended that all children and adolescents diagnosed with T1DM should be screened for hypothyroidism by means of serum TSH measurement (to identify thyroid dysfunction) and thyroid autoantibody detection [19]. Moreover, a study conducted in Turkey in 2013 on autoimmune diseases in children with T1DM, that included 1032 patients, showed that $12 \%$ of cases had chronic lymphocytic thyroiditis [29].

Patients with Down syndrome are at an increased risk of developing thyroid disease, primarily of autoimmune etiology; this has been demonstrated by many studies. A study conducted in 1978 showed that $17 \%$ of confirmed Down syndrome patients had hypothyroidism [18]. Furthermore, a study performed in 1985 showed that thyroid autoantibodies were detected in $29 \%$ of patients with Down syndrome [30]. Similarly, a Swedish study, conducted in 1997, showed that $39 \%$ of Down syndrome patients had thyroid autoantibodies [31] In the present study, $8.3 \%$ of patients with acquired hypothyroidism had Down syndrome. Some studies recommend annual screening for thyroid function in patients with Down syndrome because the symptoms of hypothyroidism might be mistaken for symptoms related to the natural course of Down syndrome [32]. This study had some limitations. Unfortunately, there were missing data that could not be obtained, either because of incomplete files or because patients were transferred to other hospitals to continue their management.

\section{Conclusion}

Acquired hypothyroidism has various clinical presentations including short stature, loss of appetite, weight gain, fatigability, constipation, cold intolerance, and goiter. physicians should have a high index of suspicion as most cases present with one isolated symptom. Prompt recognition of the findings can lead to early and effective treatment. Significantly lower levels of vitamin D were documented in patients with autoimmune thyroiditis argue for screening for vitamin D levels in patients with thyroid diseases. In our community, vitamin D supplementation is useful to decrease the prevalence of autoimmune thyroiditis.

\section{Acknowledgement}

The authors would like to thank Dr. Dareen Saleh Alghalbi, Dr. Shahad Jamil Ashgar, Dr. Arwa Ali Badakhan, Dr. Nawaf Ahmed Alfarsi, Rawan Khalid Aljaber and Maryam Abdullah Nahas for their effort in data collection. 
Citation: Al-Agha AE, Alshugair RM, Aljunedi WA, Badakhan BA (2016) Clinical Presentation of Acquired Hypothyroidism and Associated Disorders in Children and Adolescents at King Abdul-Aziz University Hospital in a Western Region of Saudi Arabia. J Pat Care 2: 123. doi: 10.4172/25734598.1000123

Page 4 of 4

\section{References}

1. LaFranchi S (2016) Acquired hypothyroidism in childhood and adolescence. UpToDate ${ }^{\circledR}$.

2. Gaitonde DY, Rowley KD, Sweeney LB (2012) Hypothyroidism: An update. South African Family Practice 2012; 54: 384-390.

3. Devdhar M, Ousman YH, Burman KD (2007) Hypothyroidism. Endocrino Metab Clin North Am 36: 595-615

4. American Diabetes Association (2012) Diagnosis and classification of diabetes mellitus. Diabetes Care 35: S64-71.

5. Cohen P, Rogol AD, Deal CL, Saenger P, Reiter EO, et al. (2008) Consensus statement on the diagnosis and treatment of children with idiopathic short stature: A summary of the growth hormone research society, the Lawson Wilkins Pediatric Endocrine Society and the European Society for Paediatric Endocrinology Workshop. J Clin Endocrinol Metab 93: 4210-4217.

6. Rallison ML, Dobyns BM, Keating FR, Rall JE, Tyler FH (1975) Occurrence and natural history of chronic lymphocytic thyroiditis in childhood. J Pediatr 86: $675-682$

7. de Vries L, Bulvik S, Phillip M (2009) Chronic autoimmune thyroiditis in children and adolescents: At presentation and during long-term follow-up. Arch Dis Child 94: 33-37.

8. Demirbilek H, Kandemir N, Gonc EN, Ozon A, Alikasifoglu A, et al. (2007) Hashimoto's thyroiditis in children and adolescents: a retrospective study on clinical, epidemiological and laboratory properties of the disease. J Pediatr Endocrinol Metab 20: 1199-1205.

9. Ozcelik T, Uz E, Akyerli CB, Bagislar S, Mustafa CA, et al (2006) Evidence from autoimmune thyroiditis of skewed $X$-chromosome inactivation in female predisposition to autoimmunity. Eur J Hum Genet 14: 791-797.

10. Ansar Ahmed S, Young PR, Penhale WJ (1983) The effects of female sex steroids on the development of autoimmune thyroiditis in thymectomized and irradiated rats. Clin Exp Immunol 54: 351-358.

11. Chowdhury S, Sarkar T, Haldar D, Taraphdar P, Naskar T, et al. (2011) Short stature in children: Experience from a tertiary care hospital in Kolkata, India. The Health 2: 139-142.

12. Gutch M, Kumar S, Razi SM, Gupta A, Kumar S, et al. (2015) Prevalence of short stature in juvenile hypothyroidism and the impact of treatment on various skeletal manifestation and growth velocity in a teritary care center. CHRISMED Journal of Health and Research 2: 251-256.

13. Reinehr T, Isa A, de Sousa G, Dieffenbach R, Andler W (2008) Thyroid hormones and their relation to weight status. Horm Res 70: 51-57.

14. Reinehr T, Andler W (2002) Thyroid hormones before and after weight loss in obesity. Arch Dis Child 87: 320-323.

15. Scott JC Jr., Mussey E (1964) Menstrual patterns in myxedema. Am J Obste Gynecol 90: 161-165.

16. Krassas GE, Pontikides N, Kaltsas T, Papadopoulou P, Paunkovic J, et al.
(1999) Disturbances of menstruation in hypothyroidism. Clin Endocrinol (Oxf) 50: 655-659.

17. Surks MI (2016) Clinical manifestations of hypothyroidism. UptoDate ${ }^{\circledR}$

18. Sare Z, Ruvalcaba RH, Kelley VC (1978) Prevalence of thyroid disorder in Down syndrome. Clin Genet 14: 154-158.

19. McKenna MJ, Herskowitz R, Wolfsdorf JI (1990) Screening for thyroid disease in children with IDDM. Diabetes Care: 801-803.

20. Baeke F, Takiishi T, Korf H, Gysemans C, Mathieu C (2010) Vitamin D Modulator of the immune system. Curr Opin Pharmacol 10: 482-496.

21. Shin DY, Kim KJ, Kim D, Hwang S, Lee EJ (2014) Low serum vitamin D is associated with anti-thyroid peroxidase antibody in autoimmune thyroiditis. Yonsei Med J 55: 476-481.

22. Kivity S, Agmon-Levin N, Zisappl M, Shapira Y, Nagy EV, et al (2011) Vitamin D and autoimmune thyroid diseases. Cell Mol Immunol 8: 243-247.

23. Mackawy AM, Al-Ayed BM, Al-Rashidi BM (2013) Vitamin D deficiency and its association with thyroid disease. Int J Health Sci (Qassim) 7: 267-275.

24. Perros P, McCrimmon RJ, Shaw G, Frier BM (1995) Frequency of thyroid dysfunction in diabetic patients: Value of annual screening. Diabet Med 12 622-627.

25. Umpierrez GE, Latif KA, Murphy MB, Lambeth HC, Stentz F, et al (2003). Thyroid dysfunction in patients with type 1 diabetes: A longitudinal study. Diabetes Care 26: 1181-1185.

26. Barker JM, Yu J, Yu L, Wang J, Miao D, et al. (2005) Autoantibody "subspecificity" in type 1 diabetes: Risk for organ-specific autoimmunity clusters in distinct groups. Diabetes Care 28: 850-855.

27. Barker JM (2006) Clinical review: Type 1 diabetes-associated autoimmunity: Natural history, genetic associations and screening. J Clin Endocrinol Metab 91: $1210-1217$.

28. Hansen D, Bennedbaek FN, Hansen LK, Hoier-Madsen M, Jacobsen BB, et al. (1999) Thyroid function, morphology and autoimmunity in young patients with insulin-dependent diabetes mellitus. Eur J Endocrinol 140: 512-518.

29. Simsek DG, Aycan Z, Ozen S, Cetinkaya S, Kara C, Abali S, et al. (2013) Diabetes care, glycemic control, complications and concomitant autoimmune diseases in children with type 1 diabetes in Turkey: A multicenter study. J Clin Res Pediatr Endocrinol 5: 20-26.

30. Loudon MM, Day RE, Duke EM. Thyroid dysfunction in Down's syndrome. Arch Dis Child 60: 1149-1151.

31. Ivarsson SA, Ericsson UB, Gustafsson J, Forslund M, Vegfors P, et al. (1997) The impact of thyroid autoimmunity in children and adolescents with Down syndrome. Acta Paediatr 86: 1065-1067.

32. Karlsson B, Gustafsson J, Hedov G, Ivarsson SA, Anneren G (1998) Thyroid dysfunction in Down's syndrome: relation to age and thyroid autoimmunity. Arch Dis Child 79: 242-245. 